\title{
Validação de indicadores para monitoramento da qualidade do pré-natal ${ }^{a}$
}

\author{
Validation of indicators for monitoring the quality of prenatal care \\ Validación de indicadores para el seguimiento de la calidad de la atención prenatal
}

\begin{abstract}
Michelle Thais Migoto ${ }^{1}$ (D) Rafael Pallisser de Oliveira ${ }^{2}$ (D) Márcia Helena de Souza Freire ${ }^{1}$ (D)

1. Universidade Federal do Paraná. Curitiba,
\end{abstract} PR, Brasil.

2. Universidade Positivo. Curitiba, PR, Brasil.
Autor Correspondente: Michelle Thais Migoto.

E-mail: michellemigoto@gmail.com

Recebido em 07/07/2021.

Aprovado em 13/09/2021.

\section{Resumo}

Objetivo: validar indicadores para o monitoramento da qualidade da assistência pré-natal. Método: estudo metodológico, com 11 especialistas da Linha de Cuidado à Saúde Materna e Infantil do Paraná, realizado em 2020. Os indicadores foram organizados em domínios de um modelo lógico e na tríade estrutura, processo e resultado. Analisado Taxa de Concordância, Razão de Validade de Conteúdo, Índice de Validade de Conteúdo e confiabilidade pelo Alfa de Cronbach. Resultados: elaboração de 35 indicadores e, após os procedimentos de validação foram readequados quanto a clareza, dois foram excluídos. Apresentaram confiabilidade excelente para clareza e relevância da estrutura $(0,94)$, do processo $(0,98)$ e do resultado $(0,94)$; bem como, em relação aos domínios do modelo lógico de entradas $(0,96)$, atividades $(0,86)$, saídas $(0,98)$, resultados $(0,86)$ e impacto $(0,96)$ Conclusão: os indicadores apresentam validade e confiabilidade para da qualidade do pré-natal, sob a ótica do monitoramento $e$ da qualidade em saúde. Implicações para a Prática: o constructo apresenta flexibilidade de aplicação para diversas dimensões territoriais como municípios, regionais de saúde e estado.

Palavras-chave: Avaliação em Saúde; Cuidado Pré-Natal; Indicadores de Qualidade em Assistência à Saúde; Política Informada por Evidências; Saúde Materno-Infantil.

\section{Abstract}

Objective: to validate indicators for monitoring the quality of prenatal care. Method: methodological study conducted in 2020 with 11 specialists of the maternal and child health care line of Paraná. The indicators were organized in domains of a logical model and in the triad structure, process, and result. The calculated agreement rate, content validity ratio, content validity index, and Cronbach's alpha reliability were analyzed. Results: 35 indicators were elaborated, which after the validation procedures were readjusted for clarity, two were excluded. It showed excellent reliability for clarity and relevance of the structure (0.94), process (0.98), and result (0.94), as well as in relation to the domains of the logical model of inputs (0.96), activities (0.86), outputs (0.98), results (0.86), and impact (0.96). Conclusion: the indicators are valid and reliable for evaluating the quality of prenata care, reflecting the impact of this care on quality management. Implications for Practice: The construct presents flexibility of application for several territorial dimensions such as municipalities, regional health, and state.

Keywords: Evidence-Informed Policy; Health Evaluation; Maternal and Child Health; Prenatal Care; Quality Indicators, Health Care.

\section{Resumen}

Objetivo: validar indicadores para el seguimiento de la calidad de la atención prenatal. Método: estudio metodológico, con 11 especialistas de la Línea de Atención Materno infantil de Paraná, indicadores organizados en dominios de un modelo lógico y en la organización de la tríada estructura, proceso y resultado, realizado en 2020. Tasa de Concordancia Calculada, Razón de Validez de Contenido, Índice de Validez de Contenido; y confiabilidad por Alfa de Cronbach. Resultados: Se elaboraron 35 indicadores, que luego de reajustar los procedimientos de validación para mayor claridad, se excluyeron dos. Mostró una excelente confiabilidad para la claridad y relevancia de la estructura (0.94), el proceso (0.98) y el resultado (0.94); así como en relación con los dominios del modelo lógico de insumos $(0,96)$, actividades $(0,86)$, productos $(0,98)$, resultados $(0,86)$ e impacto $(0,96)$. Conclusión: los indicadores son válidos y confiables para evaluar la calidad de la atención prenatal, reflejando el impacto de esta atención en la gestión de la calidad. Implicaciones para la práctica: El constructo presenta flexibilidad de aplicación para varias dimensiones territoriales como municipios, salud regional y estadual.

Palabras clave: Atención Prenatal; Evaluación en Salud; Indicadores de Calidad de la Atención de Salud; Política Informada por la Evidencia; Salud Materno-Infantil. 


\section{INTRODUÇÃO}

O cuidado pré-natal é de substancial importância para a promoção da saúde materno-infantil, tendo em vista que suas ações buscam promover a prevenção, o diagnóstico e o tratamento de doenças ou carências. Além disso, ofertar informações, sobretudo, em saúde, como também dos aspectos relativos ao suporte social, cultural e psicológico da gestante. Essas ações impactam na redução de óbitos evitáveis e melhoram a qualidade de vida da população envolvida, por aumentarem a qualidade dos cuidados de saúde ${ }^{1,2}$.

Para o fortalecimento do cuidado pré-natal no Brasil, em 2000, foi instituído o Programa de Humanização no Pré-natal e Nascimento ${ }^{3}$ que garante o acesso ao atendimento, o direito ao acompanhante e a vinculação da gestante à maternidade no momento do parto. Ademais, realizar a primeira consulta antes da $16^{\text {a }}$ semana de gestação, totalizando o mínimo de sete consultas, sendo uma delas no período puerperal. Essas consultas precisam indicar a realização dos principais exames como tipagem sanguínea, pesquisa de anemias, glicemia de jejum e teste para sífilis e HIV. Assim como oportunizar a vacinação preconizada, estabelecer a classificação do risco da gestante e, quando necessário, realizar o encaminhamento ao atendimento de alto risco no seguimento ambulatorial ou hospitalar. Essas estratégias precisam ser acompanhadas por significativa educação em saúde às gestantes ${ }^{2,3}$.

Em 2011, foi instituída a Rede Cegonha ${ }^{4}$ que organizou o Sistema Único de Saúde na lógica das Redes de Atenção, visando a melhoria do acesso, cobertura e qualidade da assistência ao pré-natal, parto, puerpério e atendimento ao recém-nascido. O prénatal é entendido como um de seus componentes, fortalecendo o atendimento preconizado e, em tempo oportuno, sobretudo, pela estratificação de risco e apoio às gestantes e disponibilidade de transporte para acesso ${ }^{2,4}$, reduzindo a mortalidade materna e infantil.

Estudo nacional, Nascer no Brasil, realizado em 2014, avaliou a qualidade do pré-natal oferecido no Sistema Único de Saúde. Identificou que $75,8 \%$ das gestantes iniciaram o acompanhamento antes da $16^{\text {a }}$ semana de gestação e $73,1 \%$ realizaram seis consultas preconizadas. $\mathrm{O}$ início tardio do pré-natal esteve relacionado a dificuldade para o diagnóstico de gravidez $(4,6 \%)$, relato de problemas pessoais $(30,1 \%)$ e barreiras de acesso $(23,2 \%)$. A dificuldade com o acesso foi identificada, especialmente, em mulheres indígenas e na região Norte. Ademais, os dados dos exames realizados e registrados na caderneta de pré-natal eram inferiores nas regiões Norte e Nordeste, tanto para exames de glicemia de jejum como para exame de ultrassonografia ${ }^{5}$.

Estudo realizado no Paraná, em 2016, avaliou a implantação da Rede Cegonha em três Regionais de Saúde, identificou que impacto positivo com a sua implantação, apresentando melhora nos indicadores de saúde materna e infantil. Identificou falhas que necessitam de estratégias de qualificação dos profissionais para os registros nos Sistemas de Informação em Saúde ${ }^{6}$. Outro estudo realizado na Regional de Saúde de Foz do Iguaçu, identificou que a qualidade do pré-natal se encontra intermediária, apesar de 81,2\% $(p<0,001)$ das gestantes realizarem seis consulta ou mais e, $79,4 \%$ $(p<0,001)$ iniciarem o acompanhamento antes de 16 semanas de gestação. Ocorrem falhas de qualidade pela ausência de consulta odontológica (72,9\%; $p<0,001)$, coleta de citologia oncótica $(62,7 \%$; $p<0,001)$ e realização de exame clínico das mamas $(42,8 \% ; p<0,001)$. Há falhas na educação em saúde pela ausência de informação explicando o risco gestacional $(59,7 \%$; $p<0,001)$, não participação em grupo de gestantes $(88.4 \% ; p<0,001)$ e ausência de visita à maternidade antes do parto $(61,6 \% ; p<0,001)^{7}$.

Para a institucionalização do monitoramento da qualidade da assistência à saúde há que se contemplar a avaliação da tríade da estrutura, do processo e do resultado. Essa necessidade é factível de ser atendida com a mensuração de indicadores, os quais favorecem a determinação das prioridades para identificação de mudanças no status da saúde causadas pelas intervenções propostas nas políticas públicas. Assim, quantificar a redução de mortes maternas e infantis evitáveis, no nível local, bem como incrementar a qualidade de vida, corrobora com os Objetivos do Desenvolvimento Sustentável e reforça a agenda global de 2016-2030. Com isso, a finalidade deste estudo foi validar indicadores para o monitoramento da qualidade da assistência pré-natal, considerando a experiência do estado do Paraná que segue as recomendações propostas pela Rede Cegonha.

\section{MÉTODO}

Trata-se de um excerto de um estudo metodológico que desenvolveu e validou um modelo lógico para a gestão sob modelo de governança, da Linha de Cuidado a Saúde Materna e Infantil, contemplando desde o pré-natal (em apresentação) até o atendimento ao recém-nascido. $O$ estudo foi desenvolvido em cinco etapas: investigação bibliográfica para seleção de evidências científicas; construção da primeira versão do instrumento; coleta de dados para validação de conteúdo; análise dos dados da validação de conteúdo; e, remodelagem do instrumento elaborado ${ }^{8}$. Para a elaboração deste estudo foi seguido as recomendações Standards for Quality Improvement Reporting Excellence (SQUIRE) ${ }^{9}$ que orienta a estrutura para o relato de novos conhecimentos sobre como melhorar os cuidados em saúde.

Na primeira etapa, foi realizada uma revisão bibliográfica ${ }^{10}$ estruturada a partir da pergunta: quais os critérios de avaliação da qualidade da assistência à saúde da mulher e da criança propostos pelas políticas públicas de saúde brasileiras? A identificação da literatura foi cotejada e apoiada por documentos legais como a Constituição Federal, leis, resoluções, portarias, instruções normativas, documentos do Ministério da Saúde, os quais orientam as políticas públicas de saúde. Esses documentos foram localizados em sites oficiais do Governo Federal, analisados e interpretados nas dimensões assistenciais, considerando-se as ações vigentes na política nacional de assistência ao pré-natal.

Para a segunda etapa, que consistiu na construção dos indicadores, relacionou-se o modelo lógico ${ }^{11} \mathrm{com}$ a tríade estrutura, processo e resultado ${ }^{12}$. O modelo lógico foi composto por cinco domínios: entradas; atividades; saídas; resultados; e, impacto, no qual a estrutura é representada pelos domínios entradas e atividades; o processo por saídas; e, os resultados por resultados e impacto ${ }^{11,12}$. Para a seleção dos indicadores, foram considerados 
os que estavam apresentados nas legislações e outros elaborados pelos autores, distribuídos conforme os domínios do modelo lógico.

O estudo foi realizado no estado do Paraná, localizado na região Sul do Brasil, composto por 399 municípios. Eles articulam seus serviços em 22 Regionais de Saúde, as quais se organizam em quatro Macrorregionais, denominadas de Leste, Norte, Noroeste e Oeste. Para o estabelecimento da ordenação dos serviços de saúde maternos e infantis, conforme recomenda a Rede Cegonha, implantada em 2012, conta-se com uma Linha de Cuidado à Saúde Materna e Infantil, na lógica das Redes de Atenção à Saúde, determinando a assistência ao pré-natal, ao parto, ao puerpério e ao recém-nascido ${ }^{13}$.

A terceira etapa, consistiu na coleta de dados para validação de conteúdo, foram incluídos profissionais com vivência na assistência, na gestão, no ensino ou na pesquisa em saúde materna e infantil. Sem restrição da área de formação e que apresentasse experiência profissional mínima de dois anos nessa linha de cuidado. Como critério de exclusão, foi considerado o não preenchimento completo do questionário de validação de conteúdo no prazo de 60 dias. Foram convidados 20 profissionais de saúde, sete se recusaram a participar e dois foram excluídos segundo o critério aplicável, totalizando 11 participantes que compuseram o Comitê de Juízes Especialistas.

Os juízes especialistas foram contactados por e-mail, mediante Carta Convite, que explicava os objetivos, a justificativa e os procedimentos de validação. Após o aceite, os participantes receberam o Termo de Consentimento Livre e Esclarecido e o formulário que foi respondido, individualmente. A coleta de dados para a validação do conteúdo aconteceu entre os meses de junho a dezembro de 2020, via on-line pela disponibilização de um link de acesso ao formulário de coleta de dados.

As respostas geraram uma base de dados que passou por um pré-processamento para correção de inadequações de digitação no preenchimento dos campos de identificação; foram excluídos dois participantes por não completarem o preenchimento do instrumento; e, por fim, a conferência das quantidades de respostas para evitar a duplicidade dos dados ou a ausência da informação. Com o préprocessamento finalizado, a base de dados foi importada para a ferramenta Software IBM SPSS que apoiou a análise dos dados.

$A$ análise de dados segue o referencial metodológico da Psicometria ${ }^{14}$ que orienta a validação de instrumentos. Foi realizada em sete passos: definição do conteúdo e sua explicação, considerando as dimensões assistenciais que envolvem a temática; a organização do modelo lógico relacionando a tríade e os domínios; a representatividade do conteúdo abordado pelos indicadores que compuseram o modelo lógico; a elaboração de uma matriz abordando as especificações da tríade, dos domínios e as descrições dos indicadores; elaboração das definições referente ao cálculo desses indicadores apresentados na matriz; análise da validade de conteúdo quanto a clareza, pertinência e abrangência, considerando a opinião do comitê de juízes especialistas; e, por fim, análise empírica da determinação dos níveis e discriminação dos indicadores.

O instrumento de coleta de dados foi estruturado em duas fase ${ }^{15}$, onde a primeira apresentou perguntas fechadas, aplicando a escala Thurstone de dois pontos: concordo e discordo. Possibilitou a realização da quarta etapa do estudo, que compreendeu a análise dos dados para a validação de conteúdo pelo cálculo da Taxa de Concordância (TC), que corresponde ao número de participantes que concordaram entre si, multiplicado por $100 \mathrm{e}$ dividido pelo número total de participantes. Na ocorrência de valores de $90 \%$ ou mais, o conteúdo foi considerado adequado, se valores abaixo, o conteúdo foi readequado, conforme as sugestões do Comitê de Juízes Especialistas ${ }^{15}$.

A seguir, foi calculada a Razão de Validade de Conteúdo (RVC), considerando o valor mínimo de 0,59 para os indicadores, representa a improvável concordância entre as respostas que ocorreram ao acaso. Quando o RVC foi inferior a 0,59 compreendese uma chance maior de $5 \%$ de concordarem ao acaso, por isso, sugerindo a exclusão do indicador ${ }^{15}$. Tanto a TC como a RVC foram analisadas quanto a clareza e pertinência dos indicadores.

A segunda fase do instrumento de coleta de dados utilizou uma escala tipo Likert de quatro pontos, a saber: 1) Não; 2) Pouco; 3) Bastante; e, 4) Muito. Esses julgamentos eram relativos à clareza e pertinência dos indicadores. Propiciou o cálculo do Índice de Validade de Conteúdo (IVC), que somou respostas "bastante" e "muito" e depois foi dividido pelo total de respostas. O valor considerado para concordância aceitável foi de 0,78 , para concordância mínima de 0,80 e, para concordância preferencial a partir de $0,90^{15}$. A seguir, foi realizada a análise da confiabilidade da consistência interna dos indicadores em relação a abrangência, segundo a tríade e os domínios pelo cálculo do Coeficiente Alfa de Cronbach. Foi considerado como confiabilidade mínima de 0,70 , medindo a correlação entre os juízes especialistas quanto à concordância, em relação aos indicadores ${ }^{15,16}$. A quinta etapa contou com remodelagem dos indicadores, seguindo as adequações, inclusão e exclusão sugeridas pelo Comitê de Juízes Especialistas.

Este estudo foi aprovado pelo Comitê de Ética em Pesquisa da Universidade Federal do Paraná, sob o Parecer Consubstanciado $\mathrm{n}^{\circ}$ 3.912.101, de 2020 .

\section{RESULTADOS}

Para a monitoramento da qualidade do pré-natal foram estabelecidos 35 indicadores que estão apresentados no Quadro 1.

Destes, 11 indicadores compuseram o domínio entradas e 9 (B.1, B.2, B.4, B.6, B.7, B.8, B.9, B.10, B.11) apresentaram TC menor que $90,0 \%$. Foram reavaliados quanto à clareza e pertinência e readequados conforme apresentado no Quadro 2. Foi calculado a RVC sobre a clareza e pertinência do indicador, a qual expressa se estão claros e são considerados como essenciais pelo comitê de juízes especialistas. Quando a RVC é maior que 0,59 é improvável que a concordância tenha acontecido ao acaso. O item B.1 apresentou RVC de 0,45 tanto para clareza como para pertinência e foi excluído (Quadro 2).

Os itens B.6, B.7 e B.8 apresentaram RVC sugestivo de exclusão quanto a clareza, mas optou-se pela adequação do indicador conforme as sugestões de alguns juízes especialistas. O indicador B.8 apresentou RVC para clareza de 0,09 e, assim, sofreu uma importante alteração em seu título e definição. Esses 
Validação de indicadores de qualidade do pré-natal

Migoto MT, Oliveira RP, Freire MHS

Quadro 1. Taxa de Concordância, Razão de Validade de Conteúdo e Índice de Validade de Conteúdo dos indicadores para a monitoramento da qualidade do pré-natal. Paraná, 2021.

\begin{tabular}{|c|c|c|c|c|c|c|}
\hline Indicadores & $\begin{array}{l}\text { TC da clareza } \\
\text { do Domínio }\end{array}$ & $\mathrm{RVC}$ & $\begin{array}{c}\text { TC da } \\
\text { pertinência } \\
\text { do Domínio }\end{array}$ & $\mathrm{RVC}$ & $\begin{array}{l}\text { IVC de } \\
\text { clareza do } \\
\text { item }\end{array}$ & $\begin{array}{l}\text { IVC de } \\
\text { pertinência } \\
\text { do item }\end{array}$ \\
\hline B.1. Profissionais de Saúde & 72,70 & 0,45 & 72,70 & 0,45 & 0,82 & 0,73 \\
\hline B.2. Profissionais de Saúde: médicos & 81,80 & 0,64 & 90,90 & 0,82 & 0,91 & 0,82 \\
\hline B.3. Profissionais de Saúde: enfermeiros obstétricos & 90,90 & 0,82 & 90,90 & 0,82 & 1,00 & 0,91 \\
\hline B.4. Profissionais de Saúde: médicos pediatras & 81,80 & 0,64 & 100,00 & 1,00 & 1,00 & 0,91 \\
\hline B.5. Profissionais de Saúde: enfermeiros pediátricos ou neonatais & 90,90 & 0,82 & 100,00 & 1,00 & 1,00 & 0,91 \\
\hline B.6. Estabelecimento de Saúde: Unidade Básica & 63,60 & 0,27 & 90,90 & 0,82 & 0,91 & 0,91 \\
\hline B.7. Estabelecimento de Saúde: Serviço de Atendimento Móvel de Urgência & 72,70 & 0,45 & 81,80 & 0,64 & 0,82 & 0,82 \\
\hline B.8. Gastos & 54,50 & 0,09 & 81,80 & 0,64 & 0,73 & 0,82 \\
\hline B.9. Despesas & 81,80 & 0,64 & 81,80 & 0,64 & 0,82 & 0,82 \\
\hline B.10. Investimentos & 81,80 & 0,64 & 90,90 & 0,82 & 0,82 & 0,82 \\
\hline B.11. Custo & 72,70 & 0,45 & 81,80 & 0,64 & 0,82 & 0,82 \\
\hline C.1. Cobertura do Sistema de Informação em Saúde para a Atenção Básica. & 100,00 & 1,00 & 100,00 & 1,00 & 0,91 & 0,91 \\
\hline C.2. Treinamento Sistema de Informação em Saúde para a Atenção Básica. & 81,80 & 0,64 & 90,90 & 0,82 & 0,82 & 0,91 \\
\hline C.3. Completude Sistema de Informação em Saúde para a Atenção Básica. & 90,90 & 0,82 & 90,90 & 0,82 & 0,82 & 0,91 \\
\hline D.1. Gestante com pré-natal & 90,90 & 0,82 & 90,90 & 0,82 & 1,00 & 1,00 \\
\hline D.2. Início precoce do pré-natal & 90,90 & 0,82 & 90,90 & 0,82 & 1,00 & 1,00 \\
\hline D.3. Gestante com risco estratificado & 90,90 & 0,82 & 81,80 & 0,64 & 1,00 & 1,00 \\
\hline D.4. Número de consultas de pré-natal & 90,90 & 0,82 & 81,80 & 0,64 & 1,00 & 1,00 \\
\hline D.5. Número de consultas de pré-natal + puerpério & 81,80 & 0,64 & 90,90 & 0,82 & 1,00 & 1,00 \\
\hline D.6. Número de consultas de pré-natal + todos os exames & 81,80 & 0,64 & 90,90 & 0,82 & 1,00 & 1,00 \\
\hline D.7. Número de consultas de pré-natal + puerpério + todos os exames & 81,80 & 0,64 & 90,90 & 0,82 & 1,00 & 1,00 \\
\hline $\begin{array}{l}\text { D.8. Número de consultas de pré-natal + puerpério + todos os Exames + } \\
\text { vacinação difteria e tétano + teste HIV }\end{array}$ & 81,80 & 0,64 & 90,90 & 0,82 & 1,00 & 1,00 \\
\hline D.9. Gestantes imunizadas com a vacina difteria e tétano. & 90,90 & 0,82 & 81,80 & 0,64 & 1,00 & 1,00 \\
\hline E.1. Sífilis congênita & 81,80 & 0,64 & 90,90 & 0,82 & 0,82 & 1,00 \\
\hline E.2. Tétano neonatal & 90,90 & 0,82 & 90,90 & 0,82 & 1,00 & 1,00 \\
\hline E.3. HIV transmissão vertical & 90,90 & 0,82 & 90,90 & 0,82 & 1,00 & 1,00 \\
\hline $\begin{array}{l}\text { E.4. Gestantes acompanhadas pelo sistema suplementar de saúde (planos } \\
\text { de saúde) }\end{array}$ & 81,80 & 0,64 & 81,80 & 0,64 & 0,73 & 0,91 \\
\hline E.5. Tratamento de aborto & 72,70 & 0,45 & 90,90 & 0,82 & 0,82 & 0,91 \\
\hline E.6. Gravidez na adolescência & 81,80 & 0,64 & 81,80 & 0,64 & 0,91 & 1,00 \\
\hline E.7. Diagnóstico precoce de anomalias congênitas & 90,90 & 0,82 & 90,90 & 0,82 & 0,91 & 1,00 \\
\hline F.1. Razão de mortalidade materna & 100,00 & 1,00 & 100,00 & 1,00 & 1,00 & 1,00 \\
\hline F.2. Taxa de mortalidade neonatal precoce & 100,00 & 1,00 & 100,00 & 1,00 & 1,00 & 1,00 \\
\hline F.3. Taxa de mortalidade neonatal tardia & 100,00 & 1,00 & 100,00 & 1,00 & 1,00 & 1,00 \\
\hline F.4. Taxa de mortalidade neonatal & 100,00 & 1,00 & 100,00 & 1,00 & 1,00 & 1,00 \\
\hline F.5. Taxa de mortalidade perinatal & 90,90 & 0,82 & 100,00 & 1,00 & 0,91 & 1,00 \\
\hline F.6. Taxa de mortalidade fetal & 100,00 & 1,00 & 100,00 & 1,00 & 0,91 & 1,00 \\
\hline F.7. Taxa de mortalidade infantil & 100,00 & 1,00 & 100,00 & 1,00 & 1,00 & 1,00 \\
\hline F.8. Taxa de aborto & 100,00 & 1,00 & 100,00 & 1,00 & 0,91 & 1,00 \\
\hline
\end{tabular}

Fonte: os autores. Siglas: Taxa de Concordância (TC); Razão de Validade de Conteúdo (RVC); Índice de Validade de conteúdo (IVC); Sistema de Informação em Saúde para a Atenção Básica (SISAB); Vírus da Imunodeficiência Humana (HIV); difteria e tétano (dT). 
Quadro 2. Adequações realizadas nos indicadores de monitoramento do pré-natal conforme as sugestões propostas pelo Comitê de Juiz Especialista. Paraná, 2021.

\begin{tabular}{|c|c|c|}
\hline Indicadores & Sugestões dos Juízes Clareza e Relevância do Item ao Domínio & Adequações \\
\hline B.1 & $\begin{array}{l}\text { - O rótulo do item não remete ao seu conteúdo para cálculo. } \\
\text { - Trabalhar, individualmente, quantificando médico ginecologista e enfermeiro obstetra. } \\
\text { - Na Atenção Primária, o médico e o enfermeiro generalista garantem o atendimento, o } \\
\text { item não contempla os outros níveis de atenção. } \\
\text { - A maioria das gestantes são de risco habitual ou intermediário e não são atendidas por } \\
\text { especialistas. }\end{array}$ & Item excluído. \\
\hline B.2 & $\begin{array}{l}\text { - A informação mais precisa seria complementar o nome do indicador para médicos } \\
\text { ginecologista e obstetras. } \\
\text { - Indicador não contempla os três níveis de atenção. }\end{array}$ & Ajustado o nome do indicador para médico ginecologista e obstetra. \\
\hline B.4 & $\begin{array}{l}\text { - Em unidades neonatais o médico precisar ser neonatologista, } \\
\text { - Indicador não contempla os três níveis de atenção. }\end{array}$ & $\begin{array}{l}\text { Ajustado o nome e a definição do indicador incluindo o médico } \\
\text { neonatologista. }\end{array}$ \\
\hline B.6 & $\begin{array}{l}\text { - Incluir as Estratégia de Saúde da Família na avaliação. } \\
\text { - Não é claro porque a linha de cuidado medir o número de unidades de atenção primária } \\
\text { à saúde. } \\
\text { - Indicador não está claro. }\end{array}$ & $\begin{array}{l}\text { Ajustado o nome para Atenção Primária a Saúde para incluir } \\
\text { todos os tipos de unidades disponíveis, e a definição do indicador } \\
\text { incluindo a palavra número (de unidades). }\end{array}$ \\
\hline B.7 & $\begin{array}{l}\text { - Descrever melhor o que precisa e, se quer encontrar. } \\
\text { - Utilizar a definição do Plano Estadual de Saúde de 2020-2023. }\end{array}$ & $\begin{array}{l}\text { Ajustado o nome para Estabelecimento de Saúde - Rede de Atenção às } \\
\text { Urgências, e a definição do indicador segundo as premissas da rede. }\end{array}$ \\
\hline B.8 & $\begin{array}{l}\text { - Texto não está claro, poderia ser substituído por custo efetivo total. } \\
\text { - Esclarecer o que se refere aquisição de despesas. } \\
\text { - Especificar as esferas de governo, considerando que o financiamento é tripartite, } \\
\text { conforme definido na Constituição federal e o Plano Estadual de Saúde de 2020-2023. }\end{array}$ & $\begin{array}{l}\text { Ajustado o nome do indicador para receitas arrecadadas para } \\
\text { financiamento da Linha de Cuidado à Saúde Materna e Infantil e sua } \\
\text { definição, especificando o desembolso por esfera de governo. }\end{array}$ \\
\hline B.9 & $\begin{array}{l}\text { - Separar os valores do financiamento por esfera de governo. } \\
\text { - O financiamento da média e da alta complexidade é de responsabilidade tripartite. } \\
\text { Assistência custeada pelo Fundo Nacional de Saúde, Fundo Estadual de Saúde e Fundos } \\
\text { Municipais de Saúde. Utilizar a definição do Plano Estadual de Saúde de 2020-2023. } \\
\text { - Considerar que o repasse ocorre por contratos a entidades filantrópicas, consórcios, } \\
\text { prefeituras e fundos municipais. } \\
\text { - Complexo para incluir em um processo avaliativo. }\end{array}$ & $\begin{array}{l}\text { Ajustado definição considerando o atendimento ambulatorial e } \\
\text { hospitalar específico a atenção obstétrica e neonatal. }\end{array}$ \\
\hline B.10 & $\begin{array}{l}\text { - O financiamento da média e da alta complexidade é de responsabilidade tripartite. } \\
\text { Utilizar a definição do Plano Estadual de Saúde de 2020-2023. } \\
\text { - Especificar o que é investido na atenção obstétrica e neonatal. }\end{array}$ & $\begin{array}{l}\text { Ajustado definição considerando investimentos para expansão da } \\
\text { assistência obstétrica e neonatal. }\end{array}$ \\
\hline B.11 & $\begin{array}{l}\text { - Ficou muito ampla a avaliação com esse item. } \\
\text { - O financiamento da média e da alta complexidade é de responsabilidade tripartite. } \\
\text { Utilizar a definição do Plano Estadual de Saúde de 2020-2023. }\end{array}$ & $\begin{array}{l}\text { Item excluído, após ter sido considerado as alterações dos itens B. } 8 \\
\text { a B.10. }\end{array}$ \\
\hline C.2 & - Especificar número de profissionais que fizeram o treinamento ou que precisariam fazer. & $\begin{array}{l}\text { Ajustado definição do item para número de profissionais que } \\
\text { receberam capacitação. }\end{array}$ \\
\hline D.5 & $\begin{array}{l}\text { - Deixar mais claro as variáveis envolvidas no cálculo. } \\
\text { - Como foi quantificado pré-natal no item anterior, manter esse indicador apenas com } \\
\text { consultas puerperais. }\end{array}$ & $\begin{array}{l}\text { Adequado o nome e a definição do indicador considerando apenas } \\
\text { puerpério. }\end{array}$ \\
\hline D.6 & $\begin{array}{l}\text { - Deixar mais claro as variáveis envolvidas no cálculo. } \\
\text { - Não ficou claro tipo de exames, incluir os exames laboratoriais e/ou de imagem. }\end{array}$ & Adequado a definição incluindo os tipos de exames. \\
\hline D.7 & $\begin{array}{l}\text { - Deixar mais claro as variáveis envolvidas no cálculo. } \\
\text { - Não ficou claro tipo de exames, incluir os exames laboratoriais e/ou de imagem. }\end{array}$ & Adequado a definição incluindo os tipos de exames. \\
\hline D.8 & $\begin{array}{l}\text { - Deixar mais claro as variáveis envolvidas no cálculo. } \\
\text { - Deixar claro, exames laboratoriais e de imagem. }\end{array}$ & Adequado a definição incluindo os tipos de exames. \\
\hline D.9 & $\begin{array}{l}\text { - Deixar mais claro as variáveis envolvidas no cálculo. } \\
\text { - Incluir imunização com Influenza, a difteria e tétano e hepatite B, que também são } \\
\text { vacinas preconizadas para a gestante. }\end{array}$ & $\begin{array}{l}\text { Adequado o nome e a definição incluindo todas as vacinas } \\
\text { preconizadas. }\end{array}$ \\
\hline E.1 & - Separar diagnóstico de sífilis dos óbitos por sífilis. & $\begin{array}{l}\text { Adequado a definição para diagnóstico de sífilis, considerando } \\
\text { apenas a Sífilis Congênita. }\end{array}$ \\
\hline E.4 & $\begin{array}{l}\text { - Deixar mais claro as variáveis envolvidas no cálculo. } \\
\text { - Denominador ajustado para nascidos vivos, com fonte SINASC. }\end{array}$ & $\begin{array}{l}\text { Adequado a definição, alterado a base de cálculo para numerador e } \\
\text { fonte CNES, e o denominador por nascidos vivos da fonte SINASC. }\end{array}$ \\
\hline E.5 & $\begin{array}{l}\text { - Relacionar com o número de gestantes. } \\
\text { - Rever clareza na definição, em relação ao cálculo. }\end{array}$ & Adequado a título e definição do item. \\
\hline E.6 & - Deixar mais claro as variáveis envolvidas no cálculo. & Adequado definição e base de cálculo por mil nascidos vivos. \\
\hline
\end{tabular}

Fonte: os autores. 
três itens apresentavam RVC adequado $(>0,59)$ quanto avaliado à pertinência do indicador em relação ao domínio, por isso, não foram excluídos (Quadro 2).

O indicador B.11 apresentou um RVC de 0,45 para clareza e 0,64 quanto a pertinência, apesar de ser pertinente optou-se por sua exclusão, pois as adequações realizadas nos itens B.8, B.9e B.10 (Quadro 2) tornaram-no redundante, havendo sugestões por parte de alguns juízes especialistas quanto a sua exclusão, pois ele estenderia o monitoramento relacionado ao financiamento.

Foi realizado o cálculo IVC também em relação a clareza e pertinência do indicador, sendo identificado apenas dois com valor inferior a 0,78 , sugerindo-o como inaceitável. $O$ indicador B. 1 apresentou IVC para pertinência de 0,73 que justificou sua exclusão, o B.8 apresentou IVC para clareza de 0,73 fundamentando a necessidade de uma importante readequação.

Dos três indicadores que avaliam o domínio Atividades, apenas do indicador C.2 apresentou TC inferior a 90,0\%, sendo readequado (Quadro 2). Foi calculado a RVC sobre a clareza do item em relação ao domínio, não havendo valores inferiores a 0,59. Não foi identificado IVC para clareza e pertinência com valores inferiores a 0,78. Esses dois domínios, Entradas e Atividade, compõem indicadores passíveis de mensurar a Estrutura da Linha de Cuidado à Saúde Materna e Infantil.

Dos nove indicadores que compõem o domínio Saídas, referemse ao monitoramento do Processo, quatro deles (D.5, D.6, D.7, D.8) apresentaram TC menor que 90,0\% para clareza e um (D.9) para pertinência (Quadro 1) que foram readequados conforme sugestão dos juízes especialistas (Quadro 2). Não houve valores de RVC inferiores a 0,59. Quanto ao IVC, para clareza e pertinência, todos os indicadores apresentaram concordância preferencial.

Tabela 1. Confiabilidade dos indicadores de monitoramento do pré-natal segundo a tríade estrutura, processo e resultado, Paraná, 2021.

\begin{tabular}{cccc}
\hline $\begin{array}{c}\text { Estatística da } \\
\text { Escala }\end{array}$ & Estrutura & Processo & Resultados \\
\hline $\begin{array}{c}\text { Coeficiente Alfa } \\
\text { de Cronbach }\end{array}$ & 0,936 & 0,98 & 0,943 \\
\hline Média & 84,18 & 68,36 & 113,09 \\
Variância & 147,164 & 41,255 & 95,291 \\
\hline Desvio Padrão & 12,131 & 6,423 & 9,762 \\
\hline
\end{tabular}

Dos sete indicadores que compõem o domínio Resultados, quatro deles (E.1, E.4, E.5, E.6) apresentaram TC menor que 90,0\% para clareza e dois deles (E.4, E.6) para pertinência, foram readequados (Quadro 2). Foi identificado RVC inferior a 0,59 apenas para o indicador E. 5 quanto a clareza $(0,45)$, como o seu RVC para pertinência foi de 0,82 (Quadro 1), optou-se por ajustar a definição do indicador em relação a sua forma de cálculo (Quadro 2) para ser mantido. Em relação ao IVC para clareza, apenas o item E.4 apresentou valor de 0,73 , mas seu IVC referente a pertinência apresentou-se elevado $(0,92)$ (Quadro 1), portanto, o indicador sofreu adequação importante e não foi excluído (Quadro 2).

Os oito indicadores que compõem o domínio Impacto apresentaram TC maior que $90,0 \%$ não precisando de adequações. Logo, para o cálculo do RVC todos apresentaram valores superior a 0,59, não indicando exclusão de indicadores. O IVC para clareza e pertinência, todos foram superiores a 0,78 , apresentaram concordância preferencial. Tanto o domínio Resultados como o Impacto, compreendem indicadores que avaliam Resultados.

Paraestimare confiabilidade da consistência interna dos indicadores propostos para o monitoramento do pré-natal foi aplicado o teste do Coeficiente Alfa de Cronbach, que estima a correlação entre as respostas dos participantes e a correlação média entre as perguntas. Os valores indicaram uma excelente confiabilidade interna para clareza e relevância dos indicadores organizados para o monitoramento da estrutura $(0,94)$, do processo $(0,98)$ e do resultado $(0,94)$ (Tabela 1$)$.

A confiabilidade da consistência interna também foi analisada quanto a clareza e relevância segundos os domínios, onde três deles apresentaram excelente confiabilidade: entradas $(0,96)$, saídas $(0,98)$ e impacto $(0,96)$. E, outros dois com boa confiabilidade: atividades $(0,86)$ e resultados $(0,86)$, atendendo a consistência interna mínima necessária (Tabela 2).

Desse modo, os indicadores foram readequados, segundo a sugestão do Comitê de Juízes Especialistas, não havendo inclusão de novos indicadores e apenas a exclusão de dois indicadores. O Quadro 3 apresenta os indicadores com a redefinição de seus códigos conforme título, definição e base de cálculo, desenvolvido nos domínios de um modelo lógico e alinhados à tríade da estrutura, processo e resultado da Qualidade em Saúde.

\section{DISCUSSÃO}

Os procedimentos de monitoramento propostos por este estudo buscam contribuir com a gestão da Rede de Atenção temática de saúde da mulher e da criança, denominada de Rede Cegonha.

Tabela 2. Confiabilidade dos indicadores de monitoramento do pré-natal segundo os domínios entradas, atividades, saídas, resultados e impactos, Paraná, 2021.

\begin{tabular}{|c|c|c|c|c|c|}
\hline Estatística da Escala & Entradas B.2 ao B.10 & Atividades C.1 ao C.3 & Saídas D.1 ao D.9 & Resultados E.1 ao E.7 & Impacto F.1 ao F.8 \\
\hline $\begin{array}{c}\text { Coeficiente Alfa de } \\
\text { Cronbach }\end{array}$ & 0,957 & 0,863 & 0,98 & 0,862 & 0,957 \\
\hline Média & 63,09 & 21,09 & 68,36 & 51,45 & 61,64 \\
\hline Variância & 125,491 & 10,891 & 41,255 & 28,673 & 26,255 \\
\hline Desvio Padrão & 11,202 & 3,3 & 6,423 & 5,355 & 5,124 \\
\hline
\end{tabular}

Fonte: os autores. 


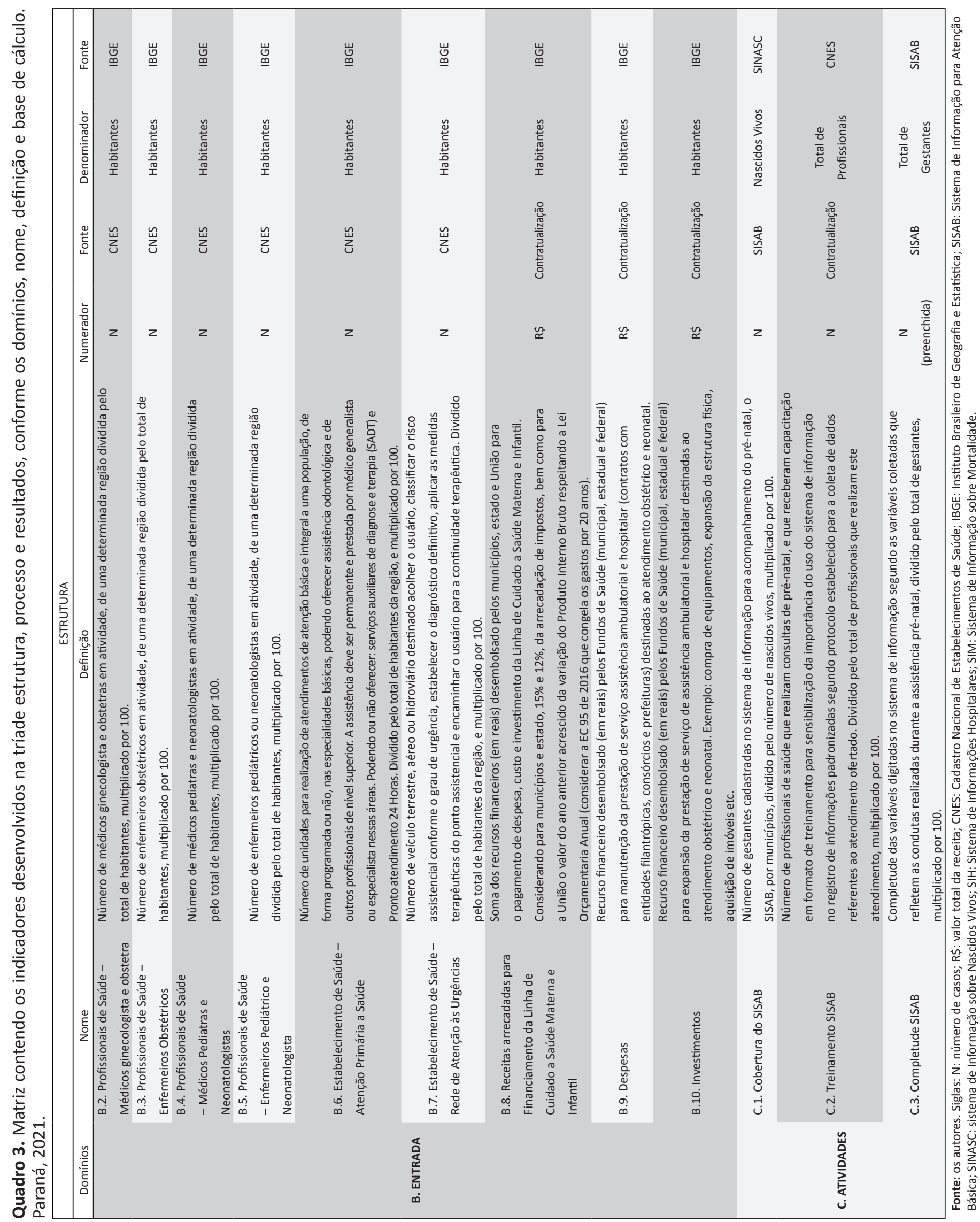




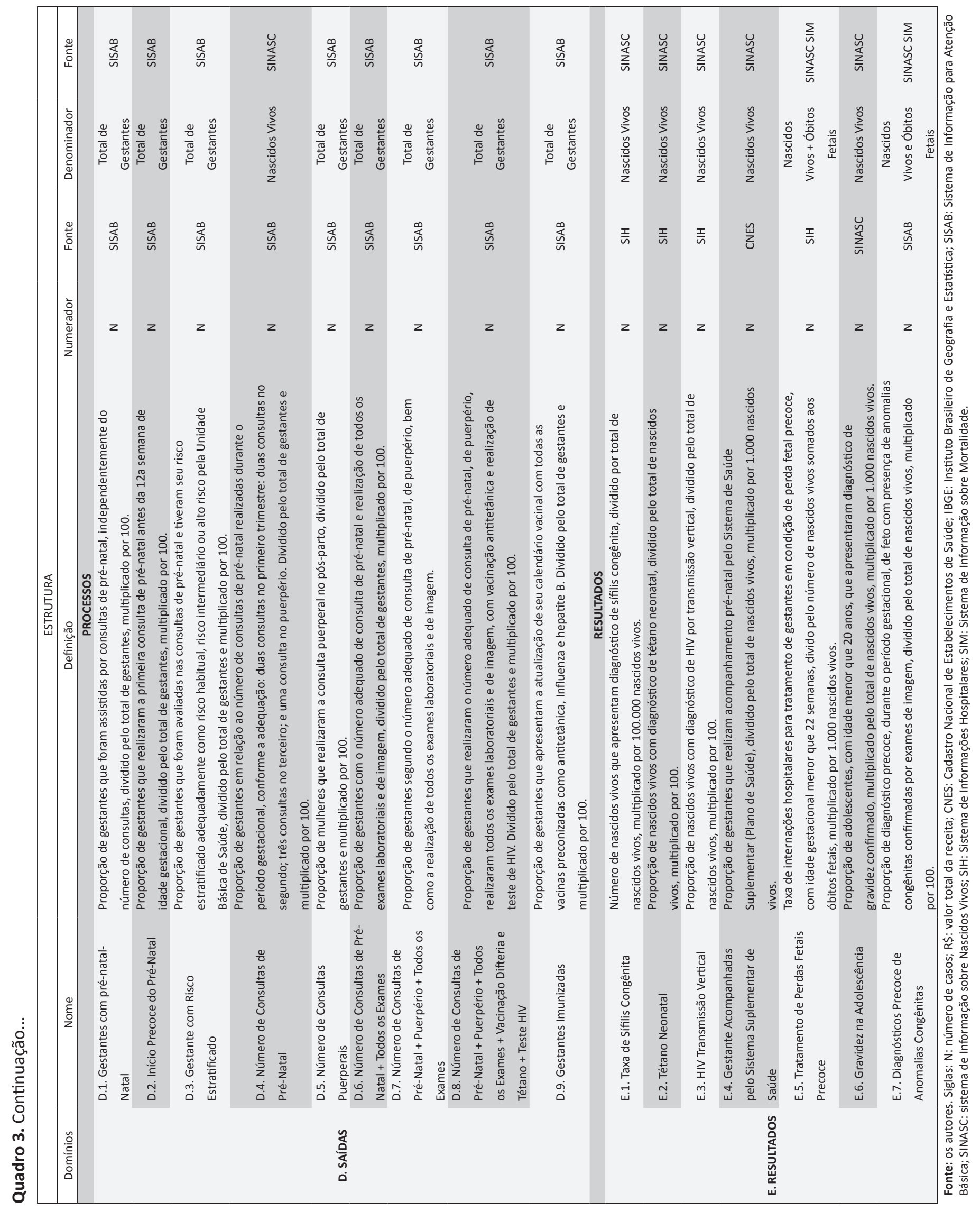




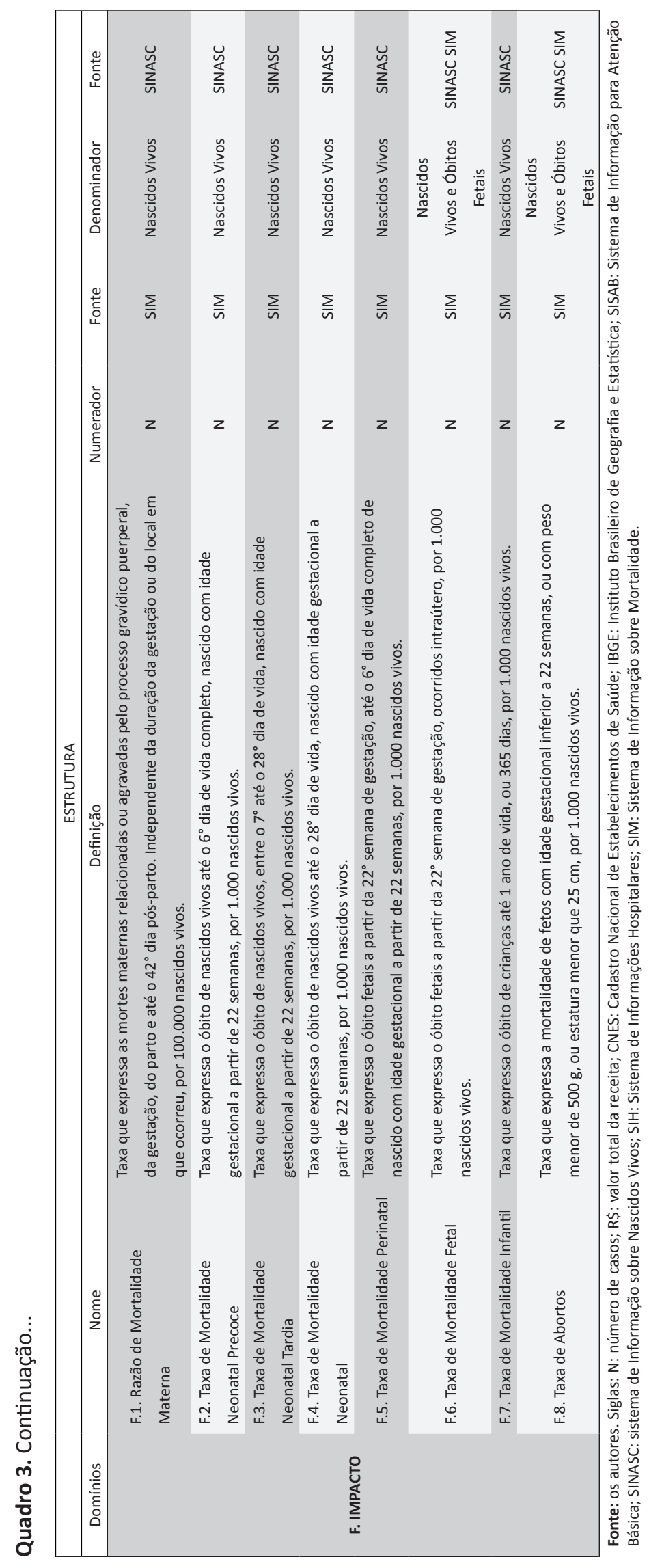


A definição de indicadores que possam mensurar a estrutura, o processo e os resultados do atendimento ao cuidado pré-natal possibilitará aos diversos atores da saúde o desenvolvimento do planejamento e o monitoramento do desempenho dos serviços e sistema de saúde ${ }^{17}$. O processo de monitoramento busca superar as fragilidades da qualidade em saúde, devido a baixa interação entre os serviços vinculados a uma rede temática, para que possa melhorar as ações de promoção à saúde e as práticas de cuidado ${ }^{18}$.

O modelo de gestão aplicado a uma Rede de Atenção envolve o conceito de governança, trata-se de uma nova organização com relação entre o Estado a suas instituições municipais, estaduais e federais, sendo elas de caráter privado, ou sem fins lucrativos, além de toda a sociedade civil. Dessa forma, a articulação entre esses diversos serviços contribui com a melhoria do acesso e da integralidade, para maior resolubilidade e humanização para a elevação da qualidade ${ }^{17} \mathrm{em}$ saúde, voltadas ao pré-natal. Assim, a aplicação dos procedimentos de monitoramento, propostos neste estudo, pode ser realizada no âmbito institucional, municipal, regional ou estadual, contribuindo para a institucionalização do monitoramento da qualidade do pré-natal.

De maneira que esse modelo de gestão consiste na governança gerencial que é organizada no nível do território ${ }^{17}$, onde as relações são estabelecidas seguindo as legislações que orientam as redes temáticas e suas prioridades de saúde, tendo a Atenção Primária como a coordenadora do cuidado ${ }^{18}$. $\mathrm{O}$ atendimento ao pré-natal, no Paraná, segue as diretrizes propostas pela Rede Cegonha, aplicadas a Linha Guia ${ }^{13}$ que é um documento, contendo definições de ações executadas na Linha de Cuidado a Saúde Materna e Infantil, evidenciando sua legitimidade e conformidade social ${ }^{19}$. Esses procedimentos de monitoramento oportunizarão a produção de evidências científicas, desde o nível local até o estadual, contribuindo ao incremento da qualidade em relação à eficácia, a efetividade, a eficiência e a otimização ${ }^{19}$.

$\mathrm{E}$, ainda, a governança gerencial visa pelo desenvolvimento de comitês que estejam conformados a uma estrutura federal para a coordenação de compromissos e prioridades de governamentais, favorecendo o diálogo entre as equipes, gestores e usuários ${ }^{18}$. Nesse diálogo, a articulação entre esses diversos atores sociais envolvidos na elaboração de uma política informada por evidências científicas corrobora para o avanço da qualidade em saúde, considerando a acessibilidade ${ }^{19}$, além de prezar pelos desejos e expectativas da população assistida na gestão e na prática profissional.

Na governança dos recursos financeiros, denominada de governança de financiamento, é realizada por meio da definição de programas que atendem a solicitação das legislações e das contratualizações entre estabelecimentos de diversos municípios ampliando o acesso ${ }^{17}$, a fim de superar a baixa capacidade gerencial e assistencial de municípios de pequeno porte ${ }^{18}$. Essa articulação fortalece as ações do atendimento pré-natal, com maior acesso e integralidade, a fim de oferecer o cuidado necessário em tempo oportuno para a redução da morbimortalidade materna e infantil. Assim, respeita o componente da equidade ${ }^{19}$ pela distribuição justa da assistência a uma sociedade, democraticamente, livre.

As evidências científicas produzidas pelos procedimentos de monitoramento, que são propostos por este estudo, apresentam como principal implicação a formulação de ações de educação permanente que subsidiarão mudanças no cuidado prestado. Tratase de uma importante ferramenta que coopera no fortalecimento do desempenho de profissionais de saúde que atuam na assistência e na gestão ${ }^{18}$ da Rede Cegonha. Permitindo a comparação entre os resultados atingidos conforme a disponibilidade de elementos de estrutura e de processo, considerando seu contexto local.

Estudo transversal, realizado em Sergipe, avaliou a qualidade do pré-natal segundo as recomendações do Programa de Humanização do Pré-Natal e Nascimento. Identificou que a cobertura foi de $99,3 \%$, sendo que $43,0 \%$ iniciaram após a $16^{\underline{a}}$ semana e $74,7 \%$ apresentavam seis consultas, conforme o preconizado pelo Ministério da Saúde, havendo significância estatística $(p<0,005)$ quanto a escolaridade com ensino médio completo, viver com o companheiro, apresentar o desejo de engravidar e estarem satisfeitas com a gestação. Quanto a qualidade dos registros de exames no cartão pré-natal apresentou-se baixa, dificultando a análise da rotina de realização exames, bem como as orientações a serem realizadas. Cerca de $64,9 \%$ dos atendimentos ocorreram na Atenção Primária, na qual 48,0\% por enfermeiros e destas 16,6\% classificadas como alto risco, onde apenas metade das mulheres foram encaminhadas ao seguimento ambulatorial de referência ${ }^{20}$.

Essas evidências científicas corroboram com os procedimentos de monitoramento propostos por este estudo. Conforme a necessidade de análise aprofundada pela aplicação de indicadores que monitorem o processo realizado no transcorrer do período pré-natal. Destaca-se que os exames preconizados se referem ao protocolo local de cada programa em execução, sugerindo a necessidade de um Sistema de Informação em Saúde que evidencie esses dados aplicáveis a uma análise para a gestão da qualidade. Entretanto, a análise de indicadores de estrutura e processo permitem o monitoramento aprofundado dos resultados de saúde, fato não relacionado no estudo transversal apresentado. Essa condição enfatiza a necessidade de um monitoramento completo que aponte os elementos da estrutura, do processo e do resultado.

\section{CONCLUSÃO}

Conclui-se que os indicadores organizados e propostos para o monitoramento da qualidade do pré-natal apresentam validade de conteúdo e confiabilidade estabelecida em conformidade com as políticas públicas de saúde. O formato desse constructo permite flexibilidade na amplitude de sua aplicação considerando as diversas dimensões territoriais, ou seja, pode ser aplicado em espaços nos quais ocorram o processo de assistência pré-natal, sejam eles restritos aos serviços de saúde, a distritos sanitários, a municípios, as regiões e macrorregiões de saúde. De modo que, em movimento de crescente ampliação e potencialidade, sua análise poderá abraçar a esfera estadual, convergindo com as recomendações da Rede Cegonha e atingir a esfera federal.

A aplicação dos procedimentos para o monitoramento favorece a sua institucionalização da avaliação, tornando-a uma prática absorvida no cotidiano das atividades dos serviços e sistema de saúde como um todo. Esse exercício é potente para suscitar a necessidade de ampliação dos indicadores e extensão de sua 
abrangência às demais dimensões assistenciais, como o parto, puerpério e atendimento ao recém-nascido.

Este estudo apresenta como limitação a ausência de participantes especialistas na área de monitoramento da qualidade da assistência, que foi compensada pela participação de especialistas em saúde pública, da criança e da mulher. Contudo, cada participante colaborou sob a ótica de sua área de conhecimento e competência profissional. Aborda-se neste estudo uma atividade complexa que pode ser concretizada com o engajamento dos diversos atores sociais envolvidos na gestão dos serviços e sistema de saúde. Outra limitação, advinda do cenário sanitário da pandemia por COVID-19 é a incipiência de diretrizes para o cuidado pré-natal às gestantes acometidas, assim, justificando a não inclusão de indicadores específicos no monitoramento.

\section{FINANCIAMENTO}

Coordenação de Aperfeiçoamento de Pessoal de Nível Superior - Brasil (CAPES) - Código de Financiamento 001, bolsa de doutorado concedido ao autor: Michelle Thais Migoto.

\section{CONTRIBUIÇÃO DOS AUTORES}

Desenho do estudo. Michelle Thais Migoto. Márcia Helena de Souza Freire

Coleta ou produção dos dados. Michelle Thais Migoto.

Análise de dados. Michelle Thais Migoto. Rafael Pallisser de Oliveira.

Interpretação dos resultados. Michelle Thais Migoto. Rafael

Pallisser de Oliveira. Márcia Helena de Souza Freire

Redação e revisão crítica do manuscrito. Michelle Thais Migoto. Rafael Pallisser de Oliveira. Márcia Helena de Souza Freire

Aprovação da versão final do artigo. Michelle Thais Migoto. Rafael Pallisser de Oliveira. Márcia Helena de Souza Freire

Responsabilidade por todos os aspectos do conteúdo e a integridade do artigo publicado. Michelle Thais Migoto. Rafael Pallisser de Oliveira. Márcia Helena de Souza Freire

\section{EDITOR ASSOCIADO}

Beatriz Rosana Gonçalves de Oliveira Toso (1)

\section{EDITOR CIENTÍFICO}

Ivone Evangelista Cabral (1)

\section{REFERÊNCIAS}

1. World Health Organization. Recommendations on antenatal care for a positive pregnancy experience. Geneva: WHO; 2016

2. Leal MD, Szwarcwald CL, Almeida PVB, Aquino EML, Barreto ML, Barros $\mathrm{F}$ et al. Saúde reprodutiva, materna, neonatal e infantil nos 30 anos do
Sistema Único de Saúde (SUS). Cien Saude Colet. 2018;23(6):1915-28. http://dx.doi.org/10.1590/1413-81232018236.03942018. PMid:29972499.

3. Portaria $n^{\circ} 569$ de 2000 , de 1 de junho de 2000 (BR). Diário Oficia da União [periódico na internet], Brasília (DF), 8 jun 2000 [citado 7 jul 2021]. Disponível em: http://bvsms.saude.gov.br/bvs/saudelegis/ gm/2000/prt0569_01_06_2000_rep.html

4. Portaria $\mathrm{n}^{\circ} 1.459$ de 2011, de 24 de junho de 2011 (BR). Institui, no âmbito do Sistema Único de Saúde - SUS - a Rede Cegonha. Diário Oficial da União [periódico na internet], Brasília (DF), 24 jun 2011 [citado 7 jul 2021]. Disponível em: http://bvsms.saude.gov.br/bvs/saudelegis/ gm/2011/prt1459_24_06_2011.html

5. Viellas EF, Domingues RMSM, Dias MAB, Gama SGN, Theme-Filha MM, Costa JV et al. Prenatal care in Brazil. Cad Saude Publica. 2014;30(Supl. 1):S85-100. http://dx.doi.org/10.1590/0102-311X00126013.PMid:25167194.

6. Frank BRB, Toso BRGO, Viera CS, Guimarães ATB, Caldeira S. Avaliação da implementação da Rede Mãe Paranaense em três Regionais de Saúde do Paraná. Saúde Debate. 2016;40(109):163-74. http://dx.doi. org/10.1590/0103-1104201610913.

7. Machado AFC, Arcoverde MAM, Caldeira S, Silva-Sobrinho RA, Silva RMM, Zilly A. Prenatal care in the perspective of the Mãe Paranaense network. Rev Norte Mineira de Enferm. 2020;1(2):78-89. http://dx.doi. org/10.46551/rnm23173092202090208.

8. Ferreira SCM, Silvino ZR, Souza DF. Pesquisa metodológica. In: Zenith RS, editor. Gestão baseada em evidência: recursos inteligentes para a solução de problemas da prática em saúde. Curitiba: Editora CRV;2018

9. Ogrinc G, Davies L, Goodman D, Batalden P, Davidoff F, Stevens D. SQUIRE 2.0: Standards for Quality Improvement Reporting Excellence: revised publication guidelines from a detailed consensus process. BMJ Qual Saf. 2016;25(12):986-92. http://dx.doi.org/10.1136/bmjqs-2015-004411. PMid:26369893.

10. Markoni MA, Lakatos EM. Fundamentos de metodologia científica. 8 ed. São Paulo: Atlas, 2017.

11. Kellogg-Foundation. Logic model development guide. 1st ed. Michigan Kellogg; 2004.

12. Aggarwal A, Aeran H, Rathee M. Quality management in healthcare: The pivotal desideratum. J Oral Biol Craniofac Res. 2019;9(2):180-2 http://dx.doi.org/10.1016/j.jobcr.2018.06.006. PMid:31211031.

13. Secretaria de Estado da Saúde do Paraná (PR). Plano Estadual de Saúde do Paraná 2016-2019 [Internet]. Curitiba: SESA; 2018 [citado 2021 jul 7]. Disponível em: http://www.saude.pr.gov.br/arquivos/File/ PlanoEstadualSaude2016MioloAlt.pdf

14. Medeiros RKS, Ferreira-Júnior MA, Pinto DPSR, Vitor AF, Santos VEP Barichello E. Pasquali's model of content validation in the Nursing research. Rev Enf Ref.2015;IV série(4):127-35. http://dx.doi.org/10.12707/RIV14009.

15. Coluci MZO, Alexandre NMC, Milani D. Construção de instrumentos de medida na área da saúde. Cien Saude Colet. 2015;20(3):925-36. http://dx.doi.org/10.1590/1413-81232015203.04332013.

16. Zanon C, Filho-Hauck N. Fidedignidade. In: Hutz CS, Baneira DR Trentini CM, editores. Psicometria. Porto Alegre: Artmed; 2015.

17. Ministério da Saúde (BR). Implantação das Redes de Atenção à Saúde e outras estratégias da SAS. Brasília: Ministério da Saúde; 2014.

18. Peiter CC, Santos JLG, Lanzoni GMM, Mello ALSF, Costa MFBNA Andrade SR. Healthcare networks: trends of knowledge development in Brazil. Esc Anna Nery. 2019;23(1):e20180214. http://dx.doi. org/10.1590/2177-9465-ean-2018-0214.

19. Bond A, Retief F, Cave B, Fundingsland M, Duinker PN, Verheem R et al A contribution to the conceptualisation of quality in impact assessment. Environ Impact Assess Rev. 2018;68:49-58. http://dx.doi.org/10.1016/j eiar.2017.10.006.

20. Mendes RB, Santos JMJ, Prado DS, Gurgel RQ, Bezerra FD, Gurgel RQ. Evaluation of the quality of prenatal care based on the recommendations Prenatal and Birth Humanization Program. Cien Saude Colet. 2020;25(3):793-804. http://dx.doi.org/10.1590/141381232020253.13182018. PMid:32159650.

\footnotetext{
a Artigo extraído da Tese de Doutorado intitulada Modelo Lógico de Avaliação da Qualidade em Saúde da Linha de Cuidado Materna e Infantil: Projeto para Gestão sob Governança, de autoria de Michelle Thais Migoto, sob a orientação da Dra. Márcia Helena de Souza Freire, desenvolvida no Programa de PósGraduação em Enfermagem da Universidade Federal do Paraná. Ano de defesa: 2021.
} 